\title{
Susceptibility of Plasmodium falciparum isolates to antimalarial drugs in a highly seasonal malaria endemic village in Mali
}

Karim Traoré ( $\boldsymbol{\nabla}$ ktraore@icermali.org)

Malaria Research and Training Center

\section{Seidina AS Diakité}

ICER/FAPH/USTTB

\section{Sekou Bah}

FAPH/USTTB

\section{Drissa S Konaté}

ICER/USTTB

Djeneba Dabitao

FAPA/UCRC/UTTB

Ibrahim Sanogo

UCRC/USTTB

Modibo Sangaré

FMOS/USTTB

Souleymane Dama

ICER/FAPH/USTTB

Bourama Keita

ICER/USTTB

Mory Doumbouya

ICER/USTTB

Merepen A Guindo

ICER/FAPH/USTTP

Mahamadou Diakité

ICER/FAPH/UCRC

\section{Research}

Keywords: Plasmodium falciparum, Ex-vivo drug sensitivity test, susceptibility, resistance, anti-malarial drugs, Mali

Posted Date: November 22nd, 2019 
DOI: https://doi.org/10.21203/rs.2.17605/v1

License: (c) (1) This work is licensed under a Creative Commons Attribution 4.0 International License. Read Full License 


\section{Abstract}

Background: In 2006, the National Malaria Control Program (NMCP) in Mali recommended artemisininbased combination therapy (ACT) as the first-line treatment for uncomplicated malaria. Since the introduction of ACT, few reports are available on the level of resistance of Plasmodium falciparum (P. falciparum) to antimalarial drugs in Mali. Dihydroartermisinin is the active metabolite of artemisinin derivatives. Here, we conducted an ex-vivo drug sensitivity testing in a rural area of southern Mali, namely the Kéniéroba village from 2016 to 2017.

Methods: Seventy-five (75) isolates of P. falciparum were successfully evaluated for ex-vivo sensitivity to key anti-malarial drugs, namely chloroquine (CQ), quinine (QN), amodiaquine (AQ), mefloquine (MQ), lumefantrine (LUM), dihydroartermisinin (DHA), and piperaquine (PPQ). P. falciparum sensitivity to these drugs was assessed using the World Wide Antimalarial Resistance Network (WWARN) SYBR-GREEN method of inhibitory concentration of $50 \%$ (IC50) determination. Reduced sensitivity to antimalarial drugs was defined as IC50 less than the WWARN standard IC50.

Results: The proportion of resistant P. falciparum isolates was $20.2 \%$ for $C Q, 40.5 \%$ for $Q N, 6.8 \%$ for $A Q$, and $1.3 \%$ for MQ. All tested P. falciparum isolates were sensitive to LUM, DHA, and PPQ. A statistically significant correlation was found between $Q N$ and AQ IC50 values $(r=0.80 ; r 2=0.64, P<0.0001)$.

Conclusions: P. falciparum isolates were sensitive to all ACT derivates tested in Kenieroba in Mali. In contrast, P. falciparum isolates were resistant to, CQ, QN, and AQ as evidenced by high IC50 to these drugs.

\section{Background}

Early treatment of a malaria episode with an efficient antimalarial drug is required to prevent lifethreatening disease outcome. Thus, the resistance of $P$. falciparum to common antimalarial drugs is a serious hurdle for malaria control in endemic countries [1]. The development of $P$. falciparum resistance to low-cost and well-tolerated antimalarial drugs such as $C Q, A Q$, antifolates, and $M Q$ [2-6] has led the World Health Organisation (WHO) and the National Malaria Control Programs (NMCP) to recommend the artemisinin-based combination therapy (ACT) for malaria treatment [7]. The rationale for using ACT drugs is 2 -folded. First, it relies on the high efficacy of artemisinin that early suppresses disease progression which could lead to life threatening manifestation. Second, there is a need to delay as long as possible the emergence and spread of artemisinin resistance worldwide. Nowadays, one of the major concerns is that we are witnessing the first signs of the emergence of parasites resistant to artemisinin derivatives in Southeast Asia [8, 9]. However, no molecular marker of such resistance has yet been identified [10]. Some of the mutations in the gene kelch (K13) have been associated with resistance to artemisinin in vitro in Asia [11-13]. Up-to-date, resistance to artemisinin has not been reported in Africa and ACTs have remained very effective $[11,14]$. However, sub-Saharan Africa remains under threat because of the widespread use of ACTs which could lead to selective pressure on ACT, and also the increasing 
intercontinental human migrations. In addition, the circulation of sub-standard or counterfeit drugs coupled to the non-adherence of patients to treatment may contribute to rapid selection of resistant malaria parasites. In 2006, the NMCP revised the treatment policy of uncomplicated malaria. Chloroquine has been replaced by two ACTs: artemether-lumefantrine (AL) and amodiaquine-artesunate (AQ-AS). For severe malaria, artesunate, artemether and QN should be used using intra-veinous route. The necessity for regular monitoring of these antimalarial resistance phenomenon is therefore essential. Ex-vivo drug susceptibility testing is one of the most efficient indirect approaches to assess the efficacy of antimalarial drugs. Few data on in-vitro and ex-vivo ACT efficacy are available from isolates circulating in Mali. Here, we report the results of an ex-vivo susceptibility of $P$. falciparum isolates to seven (7) antimalarial drugs used in Kéniéroba, Mali.

\section{Materials And Methods}

\section{P. falciparum isolates collection}

From June to October 2017, P. falciparum isolates samples were collected from malaria patients in Kéniéroba, a village located $60 \mathrm{~km}$ Southwest of Bamako, the capital of Mali on the National Road 15 in the Sudano-Guinean area of Mali (12 $2^{\circ} 50^{\prime \prime} N$ and $8^{\circ} 19^{\prime} 58^{\prime \prime}$ W). Only $P$. falciparum mono-specific malaria patients with fever were enrolled. Patients with hemolysis or antimalarial treatment or chemoprevention were not included. Around 2-5 mL of venous blood samples were collected in EDTA collection tubes after informed consent was obtained from patients or parents and/or legal guardians for children. All patients received treatment according to NMCP's recommendations.

\section{Tested anti-malarial drugs}

P. falciparum field isolates were tested against seven (7) usual malaria drugs: Chloroquine (CQ), Quinine $(\mathrm{QN})$, Mefloquine (MQ), Amodiaquine (AQ), Piperaquine (PPQ), Lumefantrine (LUM) and Dihydroartemisinin (DHA). A stock solution of CQ diphosphate, QN, MQ and DHA were prepared in 70\% ethanol. AQ and LUM were initially dissolved in methanol while PPQ was dissolved in lactic acid $0.5 \%$ first and then in DMSO. Two-fold serial dilutions were prepared using sterile distilled water and distributed in duplicate into 24-well flat-bottom plates. Final concentrations ranged from 2.44 to $2500 \mathrm{nM} / \mathrm{I}$ for CQ, 4.88 to $5000 \mathrm{nM} / \mathrm{I}$ for QN, 1.22 to $1250 \mathrm{nM} / \mathrm{I}$ for MQ, 1.22 to $1250 \mathrm{nM} / \mathrm{I}$ for l'AQ, 0.34 to $350 \mathrm{nM} / \mathrm{f}$ for LUM, 0.10 to $100 \mathrm{nM} / \mathrm{I}$ for DHA and 0.98 to $1000 \mathrm{nM} / \mathrm{I}$ for PPQ. Fifty (50) $\mu$ l of each diluted antimalarial drug were added to 96 -well plates in duplicates. The plates were dried in an open area and kept at $4^{\circ} \mathrm{C}$ for no longer than one month.

\section{Ex-vivo drug sensitivity testing of antimalarial drugs}

For ex-vivo drug sensitivity testing, spectrometry-based determination of parasite growth using SYBRGREEN (SG) method was used as described previously [15]. Briefly 2-3 $\mathrm{mL}$ of whole blood was obtained 
by venipuncture from each patient with $P$. falciparum mono-infection. The pellet from fresh blood was washed three times with incomplete RPMI 1640 medium (ICM) (Gibco ${ }^{\mathrm{TM}}$, Invitrogen Corporation, USA) buffered with $25 \mathrm{mM}$ HEPES $(5.95 \mathrm{~g})$ and centrifuged at $2000 \mathrm{rpm}$ for 5 minutes. The parasites were tested directly without culture adaptation. The suspension of parasite was distributed in 24-well plates pre-loaded with antimalarial drugs. Culture plates were incubated at $37^{\circ} \mathrm{C}$ and $5 \% \mathrm{CO}_{2}$ for 72 hours. At the end of the incubation period (which corresponds to the schizonts' stage), the plate was taken out from the incubator and frozen at $-20^{\circ} \mathrm{C}$ to read by adding SG. After, the plate was thawed for 2 hours to lyse the cells, Prepare SG lysis buffer (per plate $-10 \mathrm{~mL}$ lysis buffer, $2 \mu \mathrm{ISG}(0.002 \% \mathrm{SG})$ and use immediately. Then, $100 \mu \mathrm{l} \mathrm{SG}$ lysis buffer was added to each well. The plate was later covered with aluminum foil, shake using a plate shaker, and incubated at room temperature in the dark for 30 minutes. The amount of SG incorporated into the nucleic acids of the parasite was determined by the Fluorometer plate Reader with excitation filter of $485 \mathrm{~nm}$ and emission filter $538 \mathrm{~nm}$. The $\mathrm{IC}_{50}$, defined as a drug concentration at which the SG signal was $50 \%$ of that measured from drug-free control wells, was calculated from In-Vitro Analysis and Reporting Tool (IVART) software to fit the concentration-inhibition data. The threshold values for the reduced in ex-vivo susceptibility were as followed: $61 \mathrm{nM}, 77 \mathrm{nM}, 12$ $\mathrm{nM}, 115 \mathrm{nM}, 30 \mathrm{nM}, 135 \mathrm{nM}$ and $611 \mathrm{nM}$ for AQ, CQ, dihydroartemisin, LUM, MQ, piperaquine and QN respectively $[16,17]$.

\section{Statistical analysis}

Data were expressed as the geometric mean $\mathrm{IC}_{50}$ and $95 \%$ confidence intervals (95 Cls) were calculated after logarithmic transformation. Cross-susceptibility was analyzed using the Pearson correlation using Graphpad Prism version8. Statistical analysis of $\mathrm{IC}_{50} \mathrm{~s}$ was performed with Graphpad Prism. Two-tailed pvalues were computed and any value less than 0.05 were considered significant.

\section{Results}

In total, 75 isolates of $P$. falciparum were successfully evaluated for ex-vivo sensitivity to CQ, QN, AQ, MQ, LUM, DHA, and PPQ. Figure 1 summarizes the distribution of the tests performed and the median $\mathrm{IC}_{50}$. The QN has the highest median of IC50 with $276.1 \mathrm{nM}(123.6$ to $627.2 \mathrm{nM})$ followed by CQ with an $\mathrm{IC}_{50}$ of $42.35 \mathrm{nM}$ (34.51 to $49.45 \mathrm{nM})$, AQ with an $\mathrm{IC}_{50}$ of $27.87 \mathrm{nM}$ (21.52 to $\left.34.64 \mathrm{nM}\right)$, PPQ with an $\mathrm{IC}_{50}$ of $17.12 \mathrm{nM}(14.76$ to $18.99 \mathrm{nM}), \mathrm{MQ}$ with an $\mathrm{IC}_{50}$ of $13.53 \mathrm{nM}(12.04$ to $18.21 \mathrm{nM})$ and finally LUM with an $\mathrm{IC}_{50}$ of $11.22 \mathrm{nM}(8.84$ to $13.75 \mathrm{nM})$. In contrast the median of IC50 of DHA was the lowest at the concentration of $0.94 \mathrm{nM}$ (0.81 to $1.01 \mathrm{nM})$ (Figure 1)

The proportion of $P$. falciparum isolates with low sensitivity to antimalarial drugs were $40.5 \%$ (30/74) for QN, $20.2 \%$ (15/74) for CQ, 6.8\% (5/73) for AQ and 1.3\% (1/75) for MQ. All $P$. falciparum isolates tested showed a good sensitivity to DHA (mean IC ${ }_{50}=0.87 \mathrm{nM}(95 \%$ IC: $0.34-3.03 \mathrm{nM})$, LUM (mean IC 50 of $10.14 \mathrm{nM}(95 \%$ IC: $0.82-50.82 \mathrm{nM})$ and PPQ (mean IC ${ }_{50}=15.86 \mathrm{nM}(95 \%$ IC: $3.72-35.72 \mathrm{nM})$ Table 1. 
Regarding the correlation between $\mathrm{IC}_{50 \mathrm{~s}}$ of studied drugs, a strong correlation with statistical significance was observed between $Q N$ and $A Q I_{50}$ values $\left(r=0.80 ; r^{2}=0.64, P<0.0001\right)$ (Table2). A positive correlation was found between the ex-vivo $I_{50}$ values of $A Q$ vs $C Q\left(r=0.57 ; r^{2}=0.32, P<0.0001\right)$, CQ vs MQ $\left(r=0.29 ; r^{2}=0.08, P=0.008\right)$, CQ vs QN $\left(r=0.49 ; r^{2}=0.24, P<0.0001\right)$, DHA vs MFQ $\left(r=0.35 ; r^{2}=\right.$ $0.12, P=0.002)$, LUM vs MQ $\left(r=0.5 ; r^{2}=0.25, P<0.0001\right)$, DHA vs PPQ $\left(r=0.43 ; r^{2}=0.18, P<0.0001\right)$, DHA vs LUM $\left(r=0.30 ; r^{2}=0.09\right)$ and MQ vs PPQ $\left(r=0.45 ; r^{2}=0.20, P<0.0001\right)$. In contrast negative correlation was observed between the $\mathrm{IC}_{50}$ values of DHA vs QN $(r=-0.35, P=0.002)$, LUM vs $\mathrm{QN}(r=-$ $0.36, P=0.002)$, DHA vs $A Q(r=-0.28, P=0.01)$ and AQ vs LUM $(r=-0.45, P<0.0001)$.

\section{Discussion}

Routine assessment of antimalarial drug efficacy is one of great interest for the NMCP. Although, the molecular markers for antimalarial drug resistance are very common, cheap and affordable tool to monitor parasite resistance to antimalarial drugs. In-vivo and ex-vivo tests are necessary to confirm the resistance of parasites to a given molecule. $P$. falciparum isolates from Kenieroba were successfully evaluated for ex-vivo sensitivity to CQ, QN, AQ, MQ, LUM, DHA, and PPQ assessing their $\mathrm{IC}_{50}$. We observed higher geometric means of the $\mathrm{IC}_{50}$ for QN at $264.97 \mathrm{nM}(39.90-1567.38 \mathrm{nM})$. Similar $\mathrm{IC}_{50}$ for QNwas reported by Touré, A. O. and al. in Cote d'Ivoire in 2008 (272,12 nM) [18]. In contrast a higher $\mathrm{QN} \mathrm{IC}_{50}$ value was reported by Kwansa-Bentum, B. and al. in 2011 in Ghana with a concentration of 355,37 nM [19]. This difference with our results could be explained by the heterogeneity of the distribution of $P$. falciparum strains and the fluctuation of the phenomenon over time. Also, the proportion of QN-resistant P. falciparum isolates defined as having an $\mathrm{IC}_{50}<611 \mathrm{nM}$ reached up to $40.5 \%$ in our study. This proportion was much higher than 19.4\% (6/31) [19] in Ghana in 2011 and 9.7\% (3/31) [20] in Senegal in 2017. Sharma, S. et al. reported reduced sensiblity in 4\% of $P$. falciparum isolates in vitro to QN in 2017 in India [21]. Our result has confirmed a reduced ex-vivo sensitivity of $P$. falciparum to QN, which may guide malaria treatment policy that is recommended by the NMCP of Mali. QN is used in third-line treatment option in severe malaria and uncomplicated malaria treatment in pregnant women during the first trimester. We found a strong correlation with statistical significance between $\mathrm{IC}_{50}$ values of $\mathrm{QN}$ and $\mathrm{AQ}(\mathrm{r}$ $\left.=0.80 ; r^{2}=0.64\right)$. Cross-resistance might suggest a similar mechanism between the two antimalarial drugs.

Results of our ex-vivo susceptibility study of $P$. falciparum isolates to $\mathrm{AQ}$ obtained showed $\mathrm{IC}_{50}$ that varied between 5.73 and $88 \mathrm{nM}$ with geometric means of $25.5 \mathrm{nM}$. This result was superior to $11.2 \mathrm{nM}$ reported by Phong, NC. and al. in 2019 in Vietnam [22]. In terms of sensitivity, 6.8 \% (5/73) of isolates had reduced sensitivity to $A Q\left(\mathrm{Cl}_{50}>61 \mathrm{nM}\right)$. Diawara, S. et al. observed an increased in vitro resistance to $A Q$ with 28.1\% (9/32) in 2017 in Dakar, Sénégal [20]. Sharma, S. et al. in 2017 in Inde found 8\% reducted sensitivity to AQ [21]. Our study comfirmed the reduced sensitivity of $P$. falciparum to AQ in Kenieroba, which may quickly impact the clinical efficacy of the artemisinin combinations. 
One isolate had reduced sensitivity to MQ (1.3\%). The geometric mean of $\mathrm{IC}_{50}$ to $\mathrm{MQ}$ was $13.13 \mathrm{nM}$. The geometric mean of $\mathrm{IC}_{50}$ to $\mathrm{CQ}$ was $46.07 \mathrm{nM}$. Our result was similar to $51 \mathrm{nM}$ by Phong, NC. et al. in 2019 in Vietnam [22] and much lower than $143.94 \mathrm{nM}$ by Kaddouri, H. et al., in Bancoumana, $156.55 \mathrm{nM}$ in Faladjé and $163.76 \mathrm{nM}$ in Kollé in 2008 both conducted in Mali [23]. Our result was lower than that reported by Touré, A. O. et al. in 2008, in Ivory Coast (Abidjan) with a geometric means of 93.72 nM [18] and Nathalie W. et al. in 2014 in Dakar, Sénégal with $97.7 \mathrm{nM}$ [24]. Among the isolates tested with CQ, $20.2 \%$ (15/74) showed IC 50 values> $77 \mathrm{nM}$ (cut off resistant to CQ). On the other hand Kaddouri, H. et al., between 2004 and 2006 inMali, found a high level of resistance in vitro from P. falciparum to CQ (6069\%) [23]. Touré, A. O. et al. in 2008 in Ivory Coast found chloroquino-resistant in $26 \%(6 / 23)$ [18] ; Sharma, S. et al. in 2017 in India found a level of chloroquino-resistant of 18\% [21]. This increased sensitivity of the isolates could be related to the decreased or absent pressure on CQ. The geometric means of $\mathrm{IC}_{50}$ was $0.87 \mathrm{nM}$ for DHA, $10.14 \mathrm{nM}$ for LUM and $15.86 \mathrm{nM}$ for PPQ. All isolates tested showed a very good sensitivity to DHA, LUM, and PPQ. This supports that DHA is the ideal component of ACTs with either LUM or PPQ. This result was similar to that reported by Diawara, S. et al.,, in Sénégal [20] and Dama et al., in Mali, both in 2017 [25]. Interestingly, Fall, B.et al.,, in 2013 in Senegal observed a reduced in vitro sensitivity of $P$. falciparum to LUM with $2.9 \%$ [26]. In order to predict the different combinations possible in case of resistance to AL. We do believe that it is necessary to study the possible correlations

of the different antimalarial molecules in term of cross-resistance. The positive correlation between $\mathrm{IC}_{50}$ values ex-vivo of AQ- CQ, AQ-QN, CQ-MQ, CQ-QN, DHA-MFQ, LUM-MQ and MQ-PPQ indicates a crossresistance between these molecules.

A cross-resistance between CQ-AQ and CQ-QN and between QN-AQ has already been reported in previous studies [27-29]. A negative correlation between the $\mathrm{IC}_{50}$ values of DHA-QN, LUM-QN reflects higher activity of DHA and LUM against the isolates of $P$. falciparum QN-resistant. A negative correlation was also observed between DHA and AQ implying that they have different mechanisms of action and that isolates resistant to $A Q$ may be sensitive to DHA. This is reassuring considering the emergence of resistance to artemisinin derivatives [30-33].

In conclusion, we found that $P$. falciparum isolates from Kéniéroba had a reduced sensitivity to $Q N, A Q$, and MQ. All clinical field isolates tested showed a very good sensitivity to DHA, LUM, and PPQ. Resistance to $\mathrm{QN}$ and $\mathrm{AQ}$ showed a positive correlation between $\mathrm{IC}_{50}$ values, indicative of crossresistance between these two important anti-malarial drugs.

\section{Declarations}

\section{Ethics approval and consent to participate}

The study was approved by the ethics committee of the faculty of medicine and Pharmacy of the University of Sciences, Technics and Technologies of Bamako (USTTB), Mali. All study particiapnts signed a written consent or assent (for children) forms in order to participate to this study. 


\section{Consent for publications}

All authors read and approved the final manuscript.

\section{List of Abbreviations}

MRTC: Malaria Research and Training Center

ICER: International Center for Excellence in Research

USTTB: University of Sciences, Technics and Technologies of Bamako

ACT: Artemisinin-based Combination Therapy

WHO: World Health Organization

NMCP: National Malaria Control Program

CQ: Chloroquine

QN: Quinine

AQ: Amodiaquine

MQ: Mefloquine

LUM: Lumefantrine

DHA: Dihydroartermisinin

PPQ: Piperaquine

WWARN: World Wide Antimalarial Resistance Network

IC50: Inhibitory concentration of 50\%

\section{Competing interests}

The authors do not report a conflict of interest

\section{Funding}

This study is supported by a USTTB

\section{Author's Contributions}

Study setup; Traoré, K; Diakité, M 
Sample collection, data collection: Traoré, K ; Diakité, SAS; Sanogo, I; Konaté, D; Keita, B; Doumbouya, M

Data analysis: Traoré, K; Diakité, SAS

Manuscript writing: Traoré, $\mathrm{K}$

Manuscript review: Traoré, K; Diakité, SAS; Dabitao, D; Dama, S; Sangaré M; Bah, S; Guindo, MA; Diakité, M

\section{Acknowledgements}

We thank the parents, guardians and children who participated into this study, and the technical, clinical and nursing staff for assistance. We are grateful to many colleagues at MRTC for providing critical reviews of the manuscripts which helped improve it.

\section{References}

1. Organization, W.H., World malaria report 2015. 2015, WHO: Geneva.

2. Packard, R.M., The origins of antimalarial-drug resistance. N Engl J Med. 371(5): p. 397-9.

3. Wongsrichanalai, C., et al., Epidemiology of drug-resistant malaria. Lancet Infect Dis, 2002. 2(4): p. 209-18.

4. Spencer, H.C., Drug-resistant malaria-changing patterns mean difficult decisions. Trans R Soc Trop Med Hyg, 1985. 79(6): p. 748-58.

5. Wernsdorfer, W.H. and D. Payne, The dynamics of drug resistance in Plasmodium falciparum. Pharmacol Ther, 1991. 50(1): p. 95-121.

6. Rieckmann, K.H., D.R. Davis, and D.C. Hutton, Plasmodium vivax resistance to chloroquine? Lancet, 1989. 2(8673): p. 1183-4.

7. Organization, W.H., Guidelines for the treatment of malaria. 2011, WHO.

8. Phyo, A.P., et al., Emergence of artemisinin-resistant malaria on the western border of Thailand: a longitudinal study. Lancet. 379(9830): p. 1960-6.

9. Amaratunga, C., et al., Artemisinin-resistant Plasmodium falciparum in Pursat province, western Cambodia: a parasite clearance rate study. Lancet Infect Dis. 12(11): p. 851-8.

10. Imwong, M., et al., Exploring the contribution of candidate genes to artemisinin resistance in Plasmodium falciparum. Antimicrob Agents Chemother. 54(7): p. 2886-92.

11. Ariey, F., et al., A molecular marker of artemisinin-resistant Plasmodium falciparum malaria. Nature, 2014. 505(7481): p. 50-5.

12. Tacoli, C., et al., Artemisinin Resistance-Associated K13 Polymorphisms of Plasmodium falciparum in Southern Rwanda, 2010-2015. Am J Trop Med Hyg, 2016. 95(5): p. 1090-1093.

13. Zhang, J., et al., In vitro susceptibility of Plasmodium falciparum isolates from the China-Myanmar border area to artemisinins and correlation with $K 13$ mutations. Int J Parasitol Drugs Drug Resist, 2019. 10: p. 20-27. 
14. OMS. Rapport mondial sur l'efficacité des médicaments antipaludiques et la pharmacorésistance: 2000-2010. Genève, S.O.m.d.I.s., Rapport mondial sur l'efficacité des médicaments antipaludiques et la pharmacorésistance: 2000-2010. . Genève, Suisse: Organisation mondiale de la santé, 2011.

15. Desjardins, R.E., et al., Quantitative assessment of antimalarial activity in vitro by a semiautomated microdilution technique. Antimicrob Agents Chemother, 1979. 16(6): p. 710-8.

16. Fall, B., et al., Ex vivo susceptibility of Plasmodium falciparum isolates from Dakar, Senegal, to seven standard anti-malarial drugs. Malar J, 2011. 10: p. 310.

17. Pascual, A., et al., Multinormal in vitro distribution of Plasmodium falciparum susceptibility to piperaquine and pyronaridine. Malar J, 2015. 14: p. 49.

18. Toure, A.O., et al., [In vitro susceptibility of P. falciparum isolates from Abidjan (Cote d'Ivoire) to quinine, artesunate and chloroquine]. Sante, 2008. 18(1): p. $43-7$.

19. Kwansa-Bentum, B., et al., Plasmodium falciparum isolates from southern Ghana exhibit polymorphisms in the SERCA-type PfATPase6 though sensitive to artesunate in vitro. Malar J, 2011. 10: p. 187.

20. Diawara, S., et al., Confirmation of Plasmodium falciparum in vitro resistance to monodesethylamodiaquine and chloroquine in Dakar, Senegal, in 2015. Malar J, 2017. 16(1): p. 118.

21. Sharma, S., et al., Correlation of in vitro sensitivity of chloroquine and other antimalarials with the partner drug resistance to Plasmodium falciparum malaria in selected sites of India. Indian J Med Microbiol, 2017. 35(4): p. 485-490.

22. Phong, N.C., et al., Susceptibility of Plasmodium falciparum to artemisinins and Plasmodium vivax to chloroquine in Phuoc Chien Commune, Ninh Thuan Province, south-central Vietnam. Malar J, 2019. 18(1): p. 10.

23. Kaddouri, $\mathrm{H}_{\text {., }}$ et al., Baseline in vitro efficacy of ACT component drugs on Plasmodium falciparum clinical isolates from Mali. Int J Parasitol, 2008. 38(7): p. 791-8.

24. Wurtz, N., et al., Role of Pfmdr1 in in vitro Plasmodium falciparum susceptibility to chloroquine, quinine, monodesethylamodiaquine, mefloquine, lumefantrine, and dihydroartemisinin. Antimicrob Agents Chemother, 2014. 58(12): p. 7032-40.

25. Dama, S., et al., Reduced ex vivo susceptibility of Plasmodium falciparum after oral artemetherlumefantrine treatment in Mali. Malar J, 2017. 16(1): p. 59.

26. Fall, B., et al., Plasmodium falciparum susceptibility to anti-malarial drugs in Dakar, Senegal, in 2010: an ex vivo and drug resistance molecular markers study. Malar J, 2013. 12: p. 107.

27. Ringwald, P., et al., In vitro culture and drug sensitivity assay of Plasmodium falciparum with nonserum substitute and acute-phase sera. J Clin Microbiol, 1999. 37(3): p. 700-5.

28. Pradines, B., et al., In-vitro activity of pyronaridine and amodiaquine against African isolates (Senegal) of Plasmodium falciparum in comparison with standard antimalarial agents. J Antimicrob Chemother, 1998. 42(3): p. 333-9. 
29. Ouedraogo, J.B., et al., In vitro sensitivity of Plasmodium falciparum to halofantrine compared with chloroquine, quinine and mefloquine in the region of Bobo-Dioulasso, Burkina Faso (West Africa). Trop Med Int Health, 1998. 3(5): p. 381-4.

30. Na-Bangchang, K., et al., Declining in efficacy of a three-day combination regimen of mefloquineartesunate in a multi-drug resistance area along the Thai-Myanmar border. Malar J, 2010. 9: p. 273.

31. Woitsch, B., et al., Susceptibility to chloroquine, mefloquine and artemisinin of Plasmodium vivax in northwestern Thailand. Wien Klin Wochenschr, 2007. 119(19-20 Suppl 3): p. 76-82.

32. Noedl, $\mathrm{H}_{\text {., }}$ et al., Artemisinin resistance in Cambodia: a clinical trial designed to address an emerging problem in Southeast Asia. Clin Infect Dis, 2010. 51(11): p. e82-9.

33. Spring, M.D., et al., Dihydroartemisinin-piperaquine failure associated with a triple mutant including kelch13 C580Y in Cambodia: an observational cohort study. Lancet Infect Dis, 2015. 15(6): p. 68391.

\section{Tables}

Table 1 Ex vivo response of isolates of $P$. falciparum from Kenieroba to antimalarial drugs

\begin{tabular}{cccccccc}
\hline \multirow{2}{*}{ Drugs } & Cut-off & & & & & \\
values & \multicolumn{2}{c}{ Resistant Isolates } & \multicolumn{2}{c}{ Sensitive Isolates } & \multicolumn{2}{c}{ Total } \\
\cline { 3 - 8 } & & $\mathbf{N}$ & $\%$ & $\mathbf{N}$ & $\%$ & $\mathbf{N}$ & $\%$ \\
\hline CQ & $77 \mathrm{nM}$ & 15 & 20.2 & 59 & 79.2 & 74 & 100 \\
AQ & $61 \mathrm{nM}$ & 5 & 6.8 & 68 & 93.2 & 73 & 100 \\
MQ & $30 \mathrm{nM}$ & 1 & 1.3 & 74 & 98.7 & 75 & 100 \\
QN & $611 \mathrm{nM}$ & 30 & 40.5 & 44 & 59.5 & 74 & 100 \\
LUM & $115 \mathrm{nM}$ & 0 & 0 & 73 & 100 & 73 & 100 \\
PPQ & $135 \mathrm{nM}$ & 0 & 0 & 73 & 100 & 73 & 100 \\
DHA & $12 \mathrm{nM}$ & 0 & 0 & 69 & 100 & 69 & 100 \\
\hline
\end{tabular}

Table 2 Correlation between ex-vivo responses of isolates of $P$. falciparum from Kenieroba to anti-malarial drugs 


\begin{tabular}{llll}
\hline Drug pairing & Interpretation & $\begin{array}{l}\text { Correlation } \\
\text { Coefficient (r) }\end{array}$ & Significance value \\
& \multicolumn{3}{l}{} \\
\hline AQ vs CQ & 74 & 0.517 & $<0.0001$ \\
AQ vs DHA & 68 & -0.28 & 0.01 \\
AQ vs LUM & 73 & -0.45 & $<0.0001$ \\
AQ vs MQ & 74 & -0.04 & 0.72 \\
AQ vs PPQ & 74 & 0.07 & 0.55 \\
AQ vs QN & 73 & 0.80 & $<0.0001$ \\
CQ vs DHA & 70 & 0.096 & 0.41 \\
CQ vs LUM & 73 & 0.036 & 0.76 \\
CQ vs MQ & 76 & 0.29 & 0.008 \\
CQ vs PPQ & 75 & 0.21 & 0.06 \\
CQ vs QN & 73 & 0.49 & $<0.0001$ \\
DHA vs LUM & 67 & 0.30 & 0.012 \\
DHA vs MQ & 70 & 0.35 & 0.002 \\
DHA vs PPQ & 68 & 0.43 & $<0.0001$ \\
DHA vs QN & 67 & -0.35 & 0.002 \\
LUM vs MQ & 74 & 0.5 & $<0.0001$ \\
LUM vs PPQ & 73 & 0.11 & 0.34 \\
LUM vs QN & 71 & -0.36 & 0.002 \\
MQ vs PPQ & 75 & 0.45 & $<0.0001$ \\
MQ vs QN & 73 & -0.02 & 0.87 \\
PPQ vs QN & 72 & -0.10 & 0.37 \\
\hline
\end{tabular}

Figures 


\section{Median with $95 \% \mathrm{Cl}$}

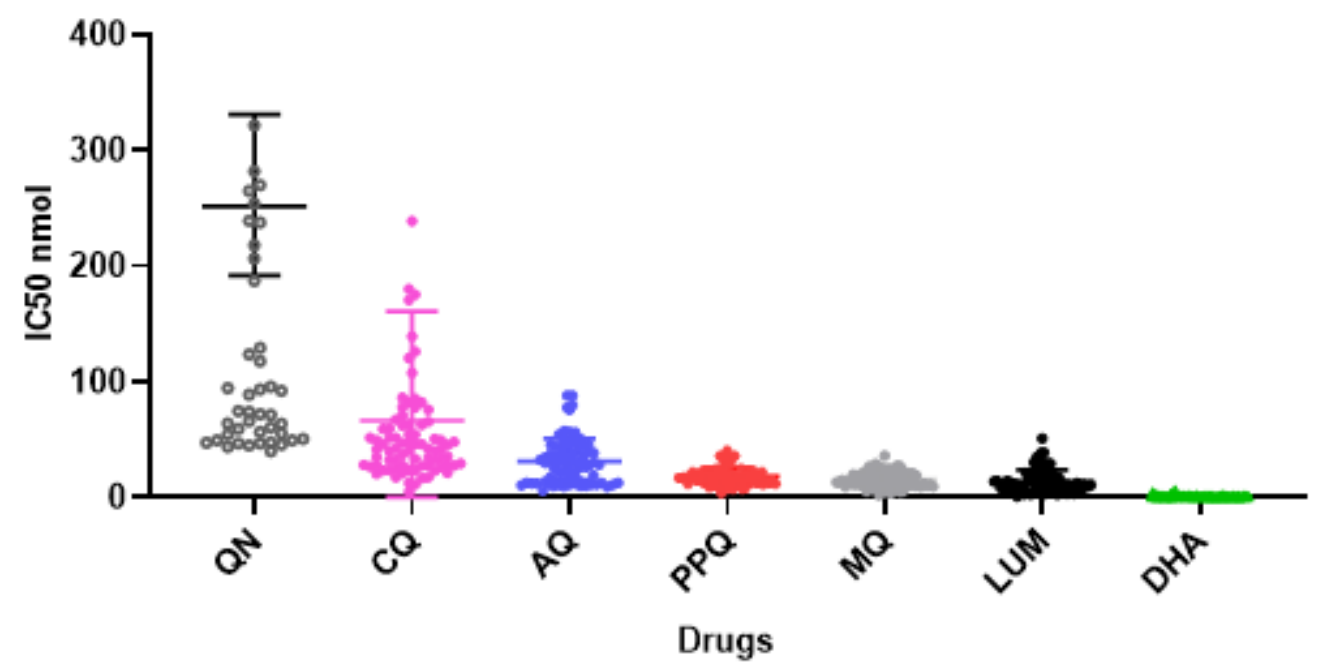

Figure 1

Median values of IC50 of different anti-malarial drugs 